\title{
The Application of Group Theory in Communication Operation Pipeline System
}

\author{
Jianqing Zhang, ${ }^{1}$ Feng Xiong $\mathbb{D},{ }^{1}$ and Jinsheng Kang $\mathbb{D}^{2}$ \\ ${ }^{1}$ School of Mechatronics Engineering and Automation, Shanghai University, Shanghai 200070, China \\ ${ }^{2}$ College of Engineering, Design and Physical Sciences, Brunel University London, London UB8 3PH, UK \\ Correspondence should be addressed to Feng Xiong; xfeng@mail.shu.edu.cn and Jinsheng Kang; jinsheng.kang@brunel.ac.uk
}

Received 27 October 2017; Accepted 22 January 2018; Published 5 March 2018

Academic Editor: Qian Zhang

Copyright (c) 2018 Jianqing Zhang et al. This is an open access article distributed under the Creative Commons Attribution License, which permits unrestricted use, distribution, and reproduction in any medium, provided the original work is properly cited.

\begin{abstract}
To resolve the "pipeline" crisis for telecom operators, this study pioneers the application of Group theory in communication operation pipeline system. The pipeline entity group model was built for information transmission in the pipeline system to analyze operation of pipeline entities. The equations of pipeline system network traffic were established according to the flux conservation principle and matrix of pipeline network. Based on pipeline entity group model, dimensionality of the matrix was reduced. The solution scheme of the flow state transition relationship of the pipeline system is obtained, which will be very useful for the telecom operators to construct high-level mobile e-commerce application model and architecture.
\end{abstract}

\section{Introduction}

With the advancement of the subversive power of mobile Internet, the new generation of information technology, such as cloud computing and Internet of things, inspired the imagination of enormous participants on the industry chain and led to a new round of information revolution in recent years. Particularly, Over-the-Top (OTT) [1-3] impacts telecom industry as a main participant of the information technology reform. Over-the-Top (OTT) refers to the phenomenon that downstream third parties of mobile Internet business chain offer a variety of valueadded telecom applications and services as an alternative to basic telecom service providers in the mobile Internet era.

To avoid the risk of marginalization and channelization, telecom service providers have to improve and guarantee the Quality of Service (QoS) in key business by constructing fine and delicacy management network and upgrading operation platform [4]. Quality of Service (QoS) can be often quantitatively measured by error rates, throughput, transmission delay, and so on $[5,6]$. Some research have been conducted to tackle this issue; for example, smart pipeline with visual, sustainable returns was proposed as a key to the telecommunication business of efficient and safe data transmission capacity $[7,8]$. Unfortunately, the proposed smart pipeline only redefined the system from two aspects of data transmission and data analysis without optimizing data traffic in distribution and transmission mechanism. Kashif et al. proposed a pipeline resource model associated with stagelevel analysis to estimate the worst-case communication latency [9], and it was only for the improvement of scheduling based on real-time analysis. Markov Decision Process (MDP) model was also used to optimize the network and improve QoS in time domain dynamically [10-13], but the established model often meets "state space explosion" problems; namely, the object management problems caused by the exponential growth of object type and quantity in the computer system and computer network make Markov Decision Process (MDP) model not able to complete the calculation accurately.

Group theory has been used widely in the field of physics and chemistry as a kind of abstract mathematical tools [14, 15]. Group theory has also been used in engineering application, for example, to reduce the multiplicative computational complexity in the creation of digital holograms [16]. In the medical image processing field, the computation time is reduced considerably by exploiting symmetry presented in breast models using Group theory in order to detect breast cancer [17]. 
Although Group theory has been used in many fields, it has never been applied to communication network before. Based on our previous work on the three-dimensional pipeline system [18] of a telecom provider in Shanghai, this study pioneers the application of Group theory on the one-dimensional pipeline content, which is the core of service quality, namely, Quality of Service (QoS). First of all, the pipeline entity of one-dimensional pipeline system is abstracted to combine the business development needs of the Internet application of the communication industry. Secondly, the pipeline system is modeled and analyzed by Group theory. Group operation of pipeline entity state is established by discrete and symmetric transformation model. Finally, the flow state transition relationship of the pipeline system is established, and the transfer process of the system state in the group operation transformation is described. The solution scheme of the flow state transition relationship of the pipeline system is given, which will be helpful for the telecom operators to construct high-level mobile e-commerce application model and architecture.

\section{Pipeline System Model Based on Group Theory}

2.1. Introduction of Pipeline Entity. In the software application level in the pipeline system, pipeline entity is a platform system of business support service, including Undertaking pipeline, Service pipeline, Payment channel, Logistics pipeline, and Data pipeline. In physics and system level, pipeline entity can connect, transport, and package information. The pipeline entity can complete all kinds of connections between objects, including payment and logistics in application and physical connection nodes. One-dimensional pipe in the pipeline system has the effects of connection, support, and transmission. It is a connected entity object and transmission of information channel. When the pipeline entity constructed to scale, pipeline system will become a platform to support higher operation output.

\subsection{Pipeline Entity Group and Pipeline Entity Object}

2.2.1. Definition of Pipeline Entity Group. Group is a set, represented by the symbol $G$. $A$ set $G$ equipped with a binary operation $\bullet$ is said to form a group $(G, \bullet)$, if the following conditions hold:

(1) $\forall a, b \in G, a \cdot b \in G$.

(2) $a \cdot(b \cdot c)=(a \cdot b) \bullet c$.

(3) There exists an element $\in G$, such that $\forall a \in G, a \bullet e=$ $e \cdot a=a$.

(4) $\forall a \in G$, there exists $a^{-1} \in G$, such that $a \cdot a^{-1}=$ $a^{-1} \cdot a=e[19]$.

The definitions of pipeline group are as follows.

Definition 1. Pipeline entity group is the set of the symmetry transformation of pipeline entity.
Definition 2. The pipeline entity element is one kind of abstract operation unit which made the pipeline status transformed, acting on the communication pipeline which is estimative and logical.

In the specified information plane, a two-dimensional plane pipeline status expressed by the pipeline and its transformation is projected to the three-dimensional information space. Similarly, the pipeline status in the information space is also projected to the pipeline entity in the real space. There is a one-to-one correlation between these transformed pipeline objects in the real space and information space. Therefore, symmetric operation and translation operation of pipeline entity are EA and CP; status change operations are listed in Table 1, for example, OP, OM, PK, PB, PS, TP, TN, UM, UD, and UC. According to the definition of group, the transformations above can be judged as a part of group elements of pipeline entity group which is 12 -order. Based on the two-dimensional nature, symmetry principle, and the symmetry transformation operations of 3-order components of information plane, the transformations can be classified into 6 types. These transformations can be defined as characteristic transformation matrixes and satisfy closure, distributive law, associative law, and inverse transformation of group. Characteristic transformation matrixes of group elements are listed in Table 1.

In the pipeline group $G$, any element $G_{i}$ or $G_{j}$ must satisfy that the result of calculation $G_{i} \bullet G_{j}$ is included in the group $G$; the symbol "•" is one kind of specific calculation, which is similar to the placeholder of integer addition. The equation of the characteristic matrix of group element can be described as follows:

$$
\begin{gathered}
a_{1}\{G 1\}+a_{2}\{G 2\}+a_{3}\{G 3, G 7, G 9\}+a_{4}\{G 4, G 8, G 10\} \\
+a_{5}\{G 5, G 11\}+a_{6}\{G 6, G 12\}=0 .
\end{gathered}
$$

Only when $a_{1}=a_{2}=a_{3}=a_{4}=a_{5}=a_{6}$ can (1) be satisfied.

Choose $G 1^{\prime}=\{G 1\}, G 2^{\prime}=\{G 2\}, G 3^{\prime}=\{G 3, G 7, G 9\}$, $G 4^{\prime}=\{G 4, G 8, G 10\}, G 5^{\prime}=\{G 5, G 11\}$, and $G 6^{\prime}=\{G 6, G 12\}$, so $G 1^{\prime}, G 2^{\prime}, G 3^{\prime}, G 4^{\prime}, G 5^{\prime}, G 6^{\prime}$ are linearly independent in the pipeline entity group; then group $G$ can be characterized by 6 order basic tensor; that is, other group elements and pipeline objects can be expressed with $G 1^{\prime}, G 2^{\prime}, G 3^{\prime}, G 4^{\prime}, G 5^{\prime}, G 6^{\prime}$. In addition, the transformations we mentioned above can only change the state of the pipeline entities, but not be related to the change of the substance of the pipeline entity.

\subsubsection{Pipeline Entity Object Status}

Definition 3. Information model of pipeline objects, $\mathrm{OM}=$ $O\{R 1, R 2, B, \ldots\}$, abbreviated as $O$.

Pipeline object $O$ is a vector and it is superposition state quantities of $R_{1}, R 2, B, \ldots$, so the operation of the pipeline entity is equivalent to the treatment of the state quantities $R 1, R 2, B, \ldots$ In the pipeline system, the pipeline state model is shown in Figure 1.

The state of $A$ at different times of the transformation is $A^{\prime}$; that is, $A^{\prime}=g A, g \in G$, where $G$ is a pipeline entity group. 
TABLE 1: Description of group elements.

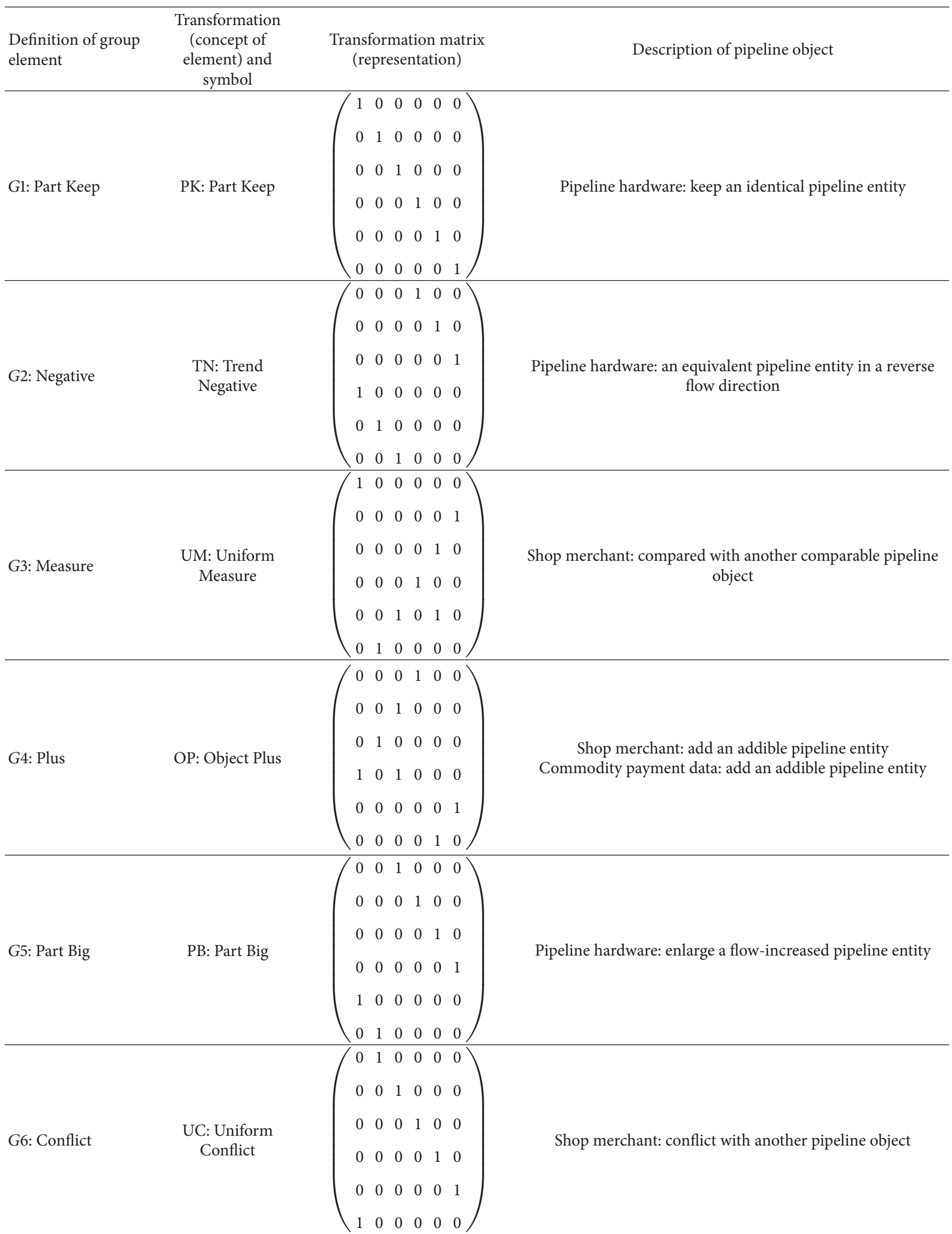


TABle 1: Continued.

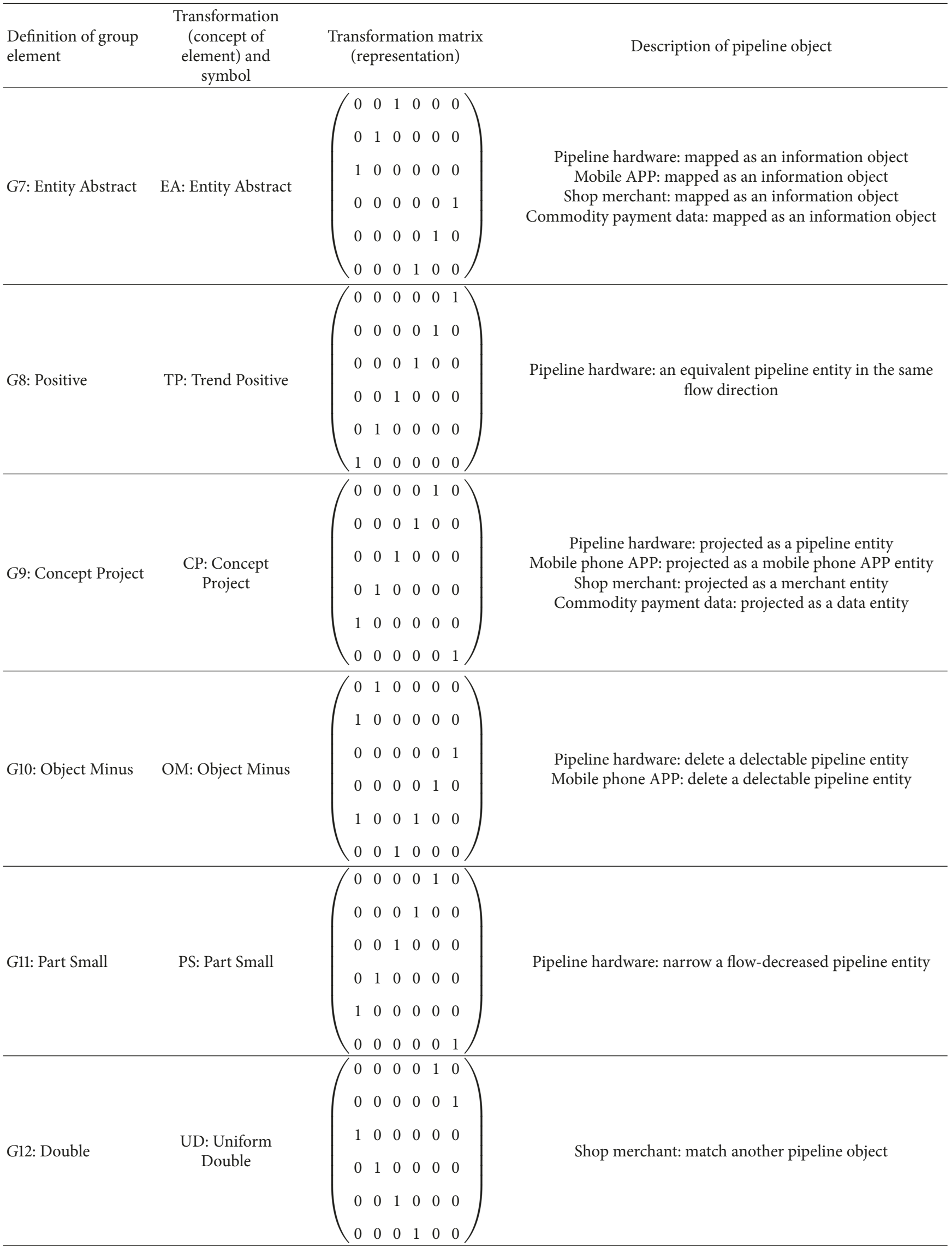




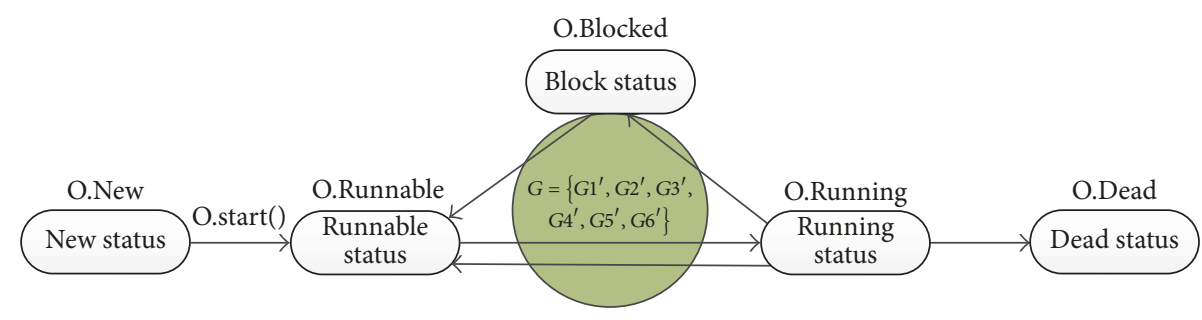

FIgURE 1: Pipeline state model.

TABLE 2: Description of state of pipeline object.

\begin{tabular}{|c|c|c|c|c|}
\hline Pipeline object & State of an object & State symbol & Associated with traffic flow & $\begin{array}{c}\text { Description of the role of } \\
\text { pipeline system }\end{array}$ \\
\hline \multirow{3}{*}{ Undertaking pipeline } & Shop building & C: construct & Generate information flow & \multirow{3}{*}{$\begin{array}{l}\text { Router, linker, } \\
\text { 2G/3G/4G/WiFi }\end{array}$} \\
\hline & Goods bearing & L: load & Generate information flow & \\
\hline & Business connection & U: union & Pass information flow & \\
\hline \multirow{2}{*}{ Service pipeline } & Service consulting & C: consult & Generate information flow & \multirow{2}{*}{$\begin{array}{l}\text { Mobile phone app, mall } \\
\text { application software }\end{array}$} \\
\hline & Service marketing & S: sale & Push information flow & \\
\hline \multirow{2}{*}{ Payment channel } & Commodity quotation & O: offer & Generate information flow & Mobile shopping mall \\
\hline & Integral payment & P: pay & Generate information flow & $\begin{array}{l}\text { Mobile phone customers, } \\
\text { rewarding system }\end{array}$ \\
\hline \multirow{2}{*}{ Logistics pipeline } & Commodity logistics access & A: add & Pass information flow & Logistics business \\
\hline & Logistics integration & L: link & Pass information flow & Commodity storage \\
\hline \multirow{3}{*}{ Data pipeline } & Data acquisition & G: gather & Generate information flow & Customers, order, delivery \\
\hline & Data summary & T: total & Pass information flow & Mall business, chart \\
\hline & $\begin{array}{l}\text { Data mining and } \\
\text { transportation }\end{array}$ & D: dig & Pass information flow & Excavating tools \\
\hline
\end{tabular}

The state of the pipeline object can be divided into ready state, running state, and blocking state in the system in which the pipeline object of the running state has an independent state. As shown in Table 2, $g$ is a group element of $G$ of the pipeline entity group, which can be expressed by the base vector as defined above:

$$
\begin{aligned}
g & =\sum_{i=1}^{6} G i^{\prime} \lambda_{i} \\
& =\left(G 1^{\prime}, G 2^{\prime}, G 3^{\prime}, G 4^{\prime}, G 5^{\prime}, G 6^{\prime}\right)\left(\begin{array}{c}
\lambda_{1} \\
\lambda_{2} \\
\vdots \\
\lambda_{6}
\end{array}\right) .
\end{aligned}
$$

2.3. Pipeline Entity Object State Transition Formula. In a realistic communication pipeline system, the state of the object is changed by the transformation operations that act on the object. It is measured in the Hilbert space of the transformation operation; in other words the state of an object is in a measurable state, which is a faithful reflection of the nature of the object. According to Definition 3 of the pipeline entity state, pipeline object in the Hilbert space is measured by the state and it is a space vector, respectively, $R 1, R 2, R 3, \ldots$ It complies with the principle of superposition in Hilbert space. According to $A^{\prime}=g A, g \in G$, and the base vector representation method, from (2) pipeline group in 6dimensional space can be described as

$$
\begin{aligned}
A^{\prime} & =\sum g_{i} A_{i} \\
& =\sum\left(G 1^{\prime}, G 2^{\prime}, G 3^{\prime}, G 4^{\prime}, G 5^{\prime}, G 6^{\prime}\right)\left(\begin{array}{c}
\lambda_{1} \\
\lambda_{2} \\
\vdots \\
\lambda_{6}
\end{array}\right) A_{i} .
\end{aligned}
$$

Therefore, similarly expanding to the pipeline object $O$, it satisfies the following formula:

$$
O^{\prime}=G * O
$$

For the pipeline entity object, $O$ represents the initial state of the pipeline object and $O^{\prime}$ represents the intrinsic vector of the object of the pipeline, which is the object state vector of the pipeline entity under the action of the transformation. From formula (3) and formula (4), the entity state transition formula can be obtained:

$$
G * O_{\text {start }}=E * O_{\text {end }} .
$$

At this point, the pipeline object state formula is established. $\mathrm{O}_{\text {start }}$ is the initial state space expression of the pipeline system 


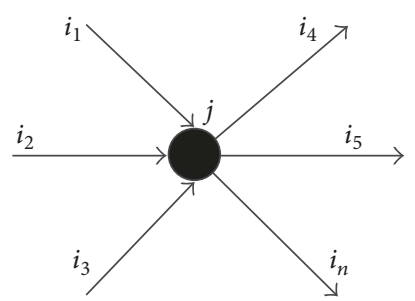

Figure 2: Model of node traffic.

and $O_{\text {end }}$ is the terminal state space expression. In the state transition formula (5), the entity pipeline is treated as a whole system. Its $O$ state changes from initial state to terminal state which is determined by the function of $G$. Among them, the quantity of flow in the transmission pipeline will not change with the change of state of pipe length, wideness, bending, and so on. The quantity of flow will not be generated or destroyed for no reason, and it stays unchanged within the pipeline transmission. This is the effect of applying Group theory on flow conservation, corresponding to reflecting the system structure of the pipeline itself. Due to the complexity of the traditional telecommunication system, the existing technology cannot provide enough storage space and reduce the throughput of the network system when the state space is too large. There are too many objects in the pipeline, which leads to the exponential increase in time complexity and the increase of time delay.

\section{The Solution of Pipeline Network Flow State}

3.1. Equations of Pipeline Network Flow. The whole pipeline network system can be seen as a system with producers and consumers; producers correspond to the service main body, providing the flow of relatively limited resources and consumers correspond to the users, utilizing producers' distribution of traffic resources. In the pipeline network model, all nodes are associated with several pipeline sections. According to the flow conservation law, the production flow is equal to the consumer flow resources of pipeline system. For any $j$ node in the model, all traffic flowing into the node should be equal to the sum of all traffic flowing out of the node, as shown in Figure 2.

There are a number of pipeline sections connected at each stage; the sections and node flow equation can be obtained as

$$
\sum_{i \in S_{j}}\left( \pm q_{i}\right)+Q_{j}=0 \quad j=1,2,3, \ldots, N
$$

where $q_{i}$ is the flow of section $i ; Q_{j}$ is the flow of node $j ; S_{j}$ is correlation sets of node $j$; and $N$ is the total number of the nodes in the network model. $\sum_{i \in S_{j}}\left( \pm q_{i}\right)$ means the sum of nodes associated with focus section, taking a minus sign when section direction is pointing to the node and the plus sign indicating departing from the node. When the traffic section flows from node, it gets plus sign. When the traffic flows into node, it gets minus sign. Simultaneous equations of all $N$ nodes in the network model can constitute node flow equations. From (6), the node flow equations in pipeline network model can be expressed as follows:

$$
A \bar{q}+\bar{Q}=\overline{0}
$$

where $A$ is the incidence matrix of network graph; $\bar{q}=$ $\left|\begin{array}{lllll}q_{1} & q_{2} & q_{3} & \cdots & q_{n}\end{array}\right|^{T}$ is the column vector of section flow; and $\bar{Q}=\left|\begin{array}{lllll}Q_{1} & Q_{2} & Q_{3} & \cdots & Q_{n}\end{array}\right|^{T}$ is the column vector of node flow.

Because a huge number of the communication pipeline system objects and connections between object complex variables influence each other, these lead to the object "state space explosion." The structure of the correlation matrix $A$ is so large that state variable cannot be solved directly. In this paper, the symmetry of pipeline entity was applied. From pipeline network information flow under the condition of conservation, pipeline network structure characteristics under the operation of transformation are studied. Therefore, equations are symmetrically simplified so that solutions of the state status can be achieved.

In the pipeline system, the total traffic inflows and outflows are equal, namely, the total flow conservation. In the process of information sending and receiving, the flow input rate is equal to the flow output rate of pipeline system. Therefore, flow is conserved at any moment; namely, the flow in the pipeline transmission balances, as shown below.

The traffic flow is assumed as a pipe object vector. According to the flow conservation equations (7) and the object state equation (5),

$$
\sum_{N} q+\sum_{M} Q=\text { Flow }=\lambda * \text { Flow }
$$

where $q$ is the output flow rate of any section (if it is in the opposite direction from the pipeline flow, it takes a negative value); $Q$ is the input flow rate of any node (if it is in the opposite direction from the pipeline flow, it takes a negative value); $N$ is the number of all sections; and $M$ is the number of all nodes.

3.2. The Model and Solution in Flow Transmission of Pipeline Network. Pipeline network consists of $M$ nodes and one node connected to $i$ pipelines with one pipeline connecting two nodes. Therefore the largest network connection status of pipeline network is formed by $M$ nodes and $M *(M-1) / 2$ pipeline sections. In the information plane, after mapping and projection operations, the model properties of the pipeline entities are as follows.

(1) Pipeline network system consists of $N$ pipeline sections and $M$ nodes.

(2) One node can be connected to multiple pipeline sections and one pipeline section can connect only two nodes.

(3) There is no intersection between the pipeline sections.

(4) As an object of pipeline transmission, information flow meets conservation law. 
Correlation matrix $A$ of pipeline network flow formula describes the $N * M$ pipeline network connection form and it can be expressed generally as follows:

$$
A=\left[\begin{array}{ccccc}
1 & \cdots & k_{1 j} & \cdots & k_{1 M} \\
k_{21} & \cdots & k_{2 j} & \cdots & k_{2 M} \\
\vdots & \cdots & \vdots & \cdots & \vdots \\
k_{i 1} & \cdots & k_{i j} & \cdots & k_{i M} \\
\vdots & \vdots & \vdots & \vdots & \vdots \\
k_{N 1} & \cdots & k_{N J} & \cdots & 1
\end{array}\right]_{N * M} .
$$

Element $k_{i j}$ indicates whether nodes $i$ and $j$ are connected or not; when $k_{i j}=0$, nodes $i$ and $j$ are connected; when $k_{i j}=1$, nodes $i$ and $j$ are not connected. Particularly when $i=j, k_{i j}=0$. For the multipoint crossover pipeline network, the properties of node and pipeline section are different, so it is difficult to define the symmetrical characteristic of the network in the two-dimensional plane. It is traditionally difficult to solve large volume pipeline network system status problems; therefore the Group theory solving steps are designed as follows.

Step 1. For pipeline network formed by nodes and pipeline sections, under pipeline entity $G$ group symmetry transformation operation, flow of pipeline network where $M$ nodes are connected to each other is transformed. According to demand response model of pipeline network in (8), pipeline network traffic incidence matrix is built and pipeline sections and nodes flow expressions are as follows.

For one pipeline section,

$$
\text { Flow }_{l}=\sum_{3} t_{l}^{i} B p s * \text { Flow }=t B p s * \text { (vector) Flow. }
$$

For $N$ pipeline sections,

$$
\sum_{N} q=\sum_{N} \text { Flow }_{l}=\sum_{N} \sum_{3} t_{l}^{i} B p s^{i} * \text { (vector) Flow } \text {. }
$$

For one node,

$$
\text { Flow }_{n}=\frac{P_{n}}{I_{n}} * \text { Flow }
$$

For $M$ nodes,

$$
\sum_{M} Q=\sum_{M} \text { Flow }_{n}=\sum_{M} \frac{P_{n}}{I_{n}} * \text { Flow. }
$$

According to (10), (11), (12), and (13), the initial flow state formula of pipeline network is as follows:

$$
\begin{aligned}
\text { (initial) Flow } & =\sum_{M} \frac{P_{n}}{I_{n}} * \text { Flow }_{n}, \\
t_{l}^{i} & =0 .
\end{aligned}
$$

Final flow state of pipeline network is as follows:

$$
\text { (final) Flow }=\sum_{N} t_{l}^{i} B p s^{i} * \text { Flow }_{l}+\sum_{M} \frac{P_{n}}{I_{n}} * \text { Flow }_{n} .
$$

When pipeline network system is in the initial and the final state, according to (14) and (15), traffic balance formula can be obtained:

$$
\sum_{N} t_{l}^{i} B p s^{i} * \text { Flow }_{l}+\sum_{M} \frac{\left(P_{n}^{1 i}-P_{n}^{0 i}\right)}{I_{n}^{i}} * \text { Flow }_{n}=0
$$

where $B p s^{i}$ Intensity $_{n}$ are the nature of the pipeline section and the node constant of pipeline network and $t_{l}^{i}, P_{n}^{1 i}$, and $P_{n}^{0 i}$ are variables of pipeline network transmission. Generally, only part of information potential of pipeline network $P_{n}^{1 i}, P_{n}^{0 i}$ is known.

Step 2. According to (16), the matrix form of the traffic demand response mode is calculated as

$$
\begin{gathered}
\left(\begin{array}{ccc}
0 & t_{1}^{i} B p s_{1}^{i}, \ldots, t_{1}^{j} B p s_{1}^{j} & t_{1}^{m} B p s_{1}^{m} \\
\vdots & \ddots & \vdots \\
t_{1}^{m} B p s_{1}^{m} & t_{1}^{j} B p s_{1}^{j}, \ldots, t_{1}^{i} B p s_{1}^{i} & 0
\end{array}\right)_{M * M}\left(\begin{array}{c}
\text { Flow }_{\text {pipeline section }} \\
\vdots \\
\text { Flow }_{\text {pipeline section }}
\end{array}\right)_{M} \\
+\left(\begin{array}{ccc}
\sum_{M} \frac{\left(P_{1}^{1}-P_{1}^{0}\right)}{I_{1}} & 0 \cdots 0 & 0 \\
\vdots & \ddots & \vdots \\
0 & 0 \cdots 0 & \sum_{M} \frac{\left(P_{M}^{1}-P_{M}^{0}\right.}{I_{M}}
\end{array}\right)_{M * M}\left(\begin{array}{c}
\text { Flow }_{\text {node }} \\
\vdots \\
\text { Flow }_{\text {node }}
\end{array}\right)_{M}=0 .
\end{gathered}
$$


Equation (17) can be simplified as follows:

$$
\begin{aligned}
& \left(\begin{array}{ccc}
0 & t_{1}^{i} B p s_{1}^{i} I_{1}, \ldots, t_{1}^{j} B p s_{1}^{j} I_{1} & t_{1}^{m} B p s_{1}^{m} I_{1} \\
\vdots & \ddots & \vdots \\
t_{1}^{m} B p s_{1}^{m} I_{M} & t_{1}^{j} B p s_{1}^{j} I_{M}, \ldots, t_{1}^{i} B p s_{1}^{i} I_{M} & 0
\end{array}\right)_{M * M} \\
& * \overline{\text { Flow }{ }_{\text {pipeline section }}} \\
& =\left(\begin{array}{ccc}
P_{1} * K & 0 \cdots 0 & 0 \\
\vdots & \ddots & \vdots \\
0 & 0 \cdots 0 & P_{M} * K
\end{array}\right)_{M * M} * \overline{\text { Flow }_{\text {node }}},
\end{aligned}
$$

where $t_{1}^{i}$ is pipeline section's effective transmission time; $B p s_{1}^{i}$ is pipeline section's rate of information transmission; $I_{1}$ is the first node capacity; $P_{1}$ is the first throughput traffic; $K$ is ratio of pipeline section to node flow direction; and $\overline{\text { Flow }_{\text {pipeline section }}}$ and $\overline{\text { Flow }_{\text {node }}}$ are pipeline sections and node vector units.

Step 3. Transformation matrix of pipeline network $G_{M}$ is as follows:

$$
\begin{aligned}
& G_{M} \\
& =\left(\begin{array}{ccc}
0 & t_{1}^{i} B p s_{1}^{i} I_{1}, \ldots, t_{1}^{j} B p s_{1}^{j} I_{1} & t_{1}^{m} B p s_{1}^{m} I_{1} \\
\vdots & \ddots & \vdots \\
t_{1}^{m} B p s_{1}^{m} I_{M} & t_{1}^{j} B p s_{1}^{j} I_{M}, \ldots, t_{1}^{i} B p s_{1}^{i} I_{M} & 0
\end{array}\right)_{M * M},
\end{aligned}
$$

$M-1 \leq N \leq M(M-1) / 2$, where $N$ is the number of pipeline sections connected to a node. According to traffic balance formula, due to the fact that transmission time and node of the potential difference are a variation of transmission pipeline system, the information transfer rate, information potential strength of pipeline structure characteristics, and entity group $G$ are known, which makes the pipeline network form $G_{M}$ matrix; suppose that $M$ nodes and $N$ pipeline sections consist of pipeline network. Under $G$ group operation, pipeline sections and nodes connect to a network, and $G_{M}$ matrix is constructed to represent pipeline network.

$M$ nodes connected to a pipeline network are represented by the matrix $G_{M}$ above. Generally, according to (19), the actual pipeline network system can be described by $G_{M}$

$$
\begin{aligned}
& G_{M \text { general }} \\
& =\left(\begin{array}{ccc}
0 & t_{1}^{i} B p s_{1}^{i} I_{1}, \ldots, t_{1}^{j} B p s_{1}^{j} I_{1} & t_{1}^{m} B p s_{1}^{m} I_{1} \\
\vdots & \ddots & \vdots \\
t_{1}^{m} B p s_{1}^{m} I_{M} & t_{1}^{j} B p s_{1}^{j} I_{M}, \ldots, t_{1}^{i} B p s_{1}^{i} I_{M} & 0
\end{array}\right)_{M * M} .
\end{aligned}
$$

According to group theory irreducible representation method, under the condition of pipeline network flow conservation, $\left\{G_{M}\right\}$ group homomorphism in pipeline entity group $G$ group: $\left\{g_{i}\right\},\left\{G_{M}\right\} \sim G$ group. So the network structure has nothing to do with the number of nodes. Network structure depends upon three inherent characteristics: information transformation rate $B p s$, potential strength (information capacity) intensity $(I)$, and transfer duty cycle time $(t)$.

Therefore, $G_{M}$ can be expressed in a linear combination of the 6-dimensional spaces largely. $G_{M}$ matrix is defined by node flow, Flow $_{1}$, Flow $_{2}, \ldots$, Flow $_{M}$, while $G$ group is defined by group transformation, $f_{1}, f_{2}, f_{3}, f_{4}, f_{5}, f_{6}$. Transformation matrix homomorphism in pipeline entity group: $G$ group: $\left\{g_{i}\right\}$.

Step 4. Six base vectors of pipeline entity $G$ group are as follows:

$$
\begin{aligned}
G 1^{\prime} & =\{G 1\}, \\
G 2^{\prime} & =\{G 2\}, \\
G 3^{\prime} & =\{G 3, G 7, G 9\}, \\
G 4^{\prime} & =\{G 4, G 8, G 10\}, \\
G 5^{\prime} & =\{G 5, G 11\}, \\
G 6^{\prime} & =\{G 6, G 12\} .
\end{aligned}
$$

In the pipeline entity group, $G 1^{\prime}, G 2^{\prime}, G 3^{\prime}, G 4^{\prime}, G 5^{\prime}, G 6^{\prime}$ are linearly independent; according to characterization of group elements matrix, there is a set of real numbers, $a_{1}, a_{2}, a_{3}, a_{4}, a_{5}, a_{6}$, which makes the equation below hold:

$$
\begin{aligned}
G_{M} & =a_{1} G 1^{\prime}+a_{2} G 2^{\prime}+a_{3} G 3^{\prime}+a_{4} G 4^{\prime}+a_{5} G 5^{\prime} \\
& +a_{6} G 6^{\prime} .
\end{aligned}
$$

According to Step 1, $a_{1}, a_{2}, a_{3}, a_{4}, a_{5}, a_{6}$ can be calculated; according to $G 1^{\prime}, G 2^{\prime}, G 3^{\prime}, G 4^{\prime}, G 5^{\prime}, G 6^{\prime}, t_{1}, t_{2}, t_{3}, \ldots, t_{n}$ of $G_{M}$ are worked out.

Above is the process of applying Group theory to the information pipeline system. Regardless of the number of nodes and the number of pipeline sections, the $G_{M}$ matrix can be simplified to a 6-order matrix. Thus "state space explosion" problem of pipeline network can be solved and it is a universal solution.

\section{The Application of Pipeline Network System}

In pipeline network system under the application of pipeline entity group, when the flow changes in pipeline entity, changing flow behavior of the pipeline system can be described as the Application Layer, Business Object Layer, and the Network Node Layer, as shown in Figure 3.

Application Layer. A functional unit in the Application Layer is the performance behavior of pipeline entity, which is the initiator of flow changing behavior. When a certain task is 


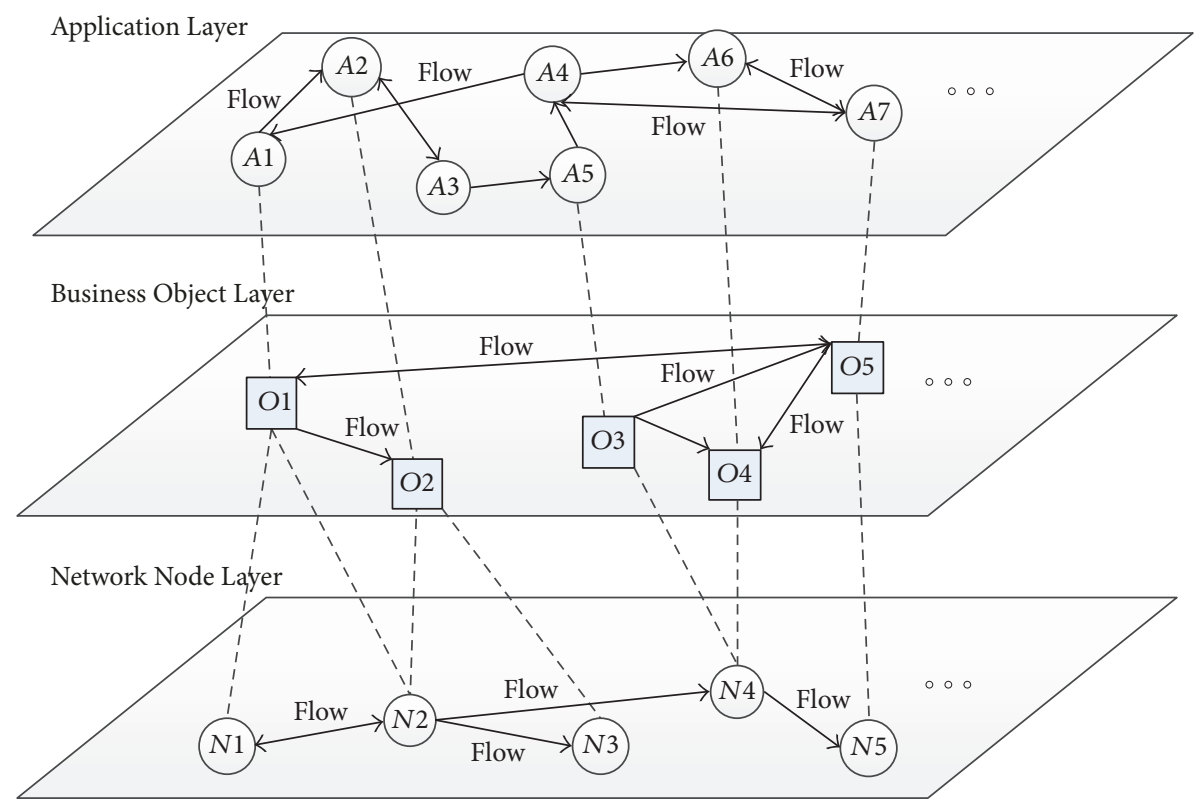

FIGURE 3: Architecture of pipeline system.

executed, traffic flows from units $A_{i}$ to $A_{j}$, to complete the business process of information transmission and exchange.

Business Object Layer. The object unit of the Business Object Layer is the abstract components of pipeline entity. When the Application Layer initiates some functional behavior, business object unit is packaging and transporting the flow, which is designated as $O_{i}$ receives and sends information to $O_{j}$.

Network Node Layer. Network Node Layer node unit consists of the physical nodes in pipeline system. When the Application Layer initiates some functional behaviors, Business Object Layer sends and receives information. In the meantime, Network Node Layer finds the optimal solution about the critical path and completes the distribution and reception of information flow.

\section{Conclusion}

In order to support the increasingly complex service quality of telecom operation system, this paper sets up the pipeline entity operation group and pipeline entity object state formula according to the current situation of mobile Internet. According to the law of flow conservation of pipeline system, the flow equation of pipe network, and pipeline object state transfer matrix, the time variables of the pipe network matrix $G_{M}$ are calculated through the linear combination of six basis vectors of the operation group. This paper proposes a new optimization method for the general information network system, which provides a new idea for the future information network system optimization.

\section{Conflicts of Interest}

The authors declare that there are no conflicts of interest regarding the publication of this paper.

\section{References}

[1] L. Ware, "Over-the-top video delivery and consumption in 2012 and beyond," SMPTE Motion Imaging Journal, vol. 121, no. 6, pp. 73-76, 2012.

[2] J. Rückert, J. Blendin, and D. Hausheer, "Software-Defined Multicast for Over-the-Top and Overlay-based Live Streaming in ISP Networks," Journal of Network and Systems Management, vol. 23, no. 2, pp. 280-308, 2015.

[3] N. Bouten, S. Latré, W. Van De Meerssche et al., "A multicastenabled delivery framework for QoE assurance of over-the-top services in multimedia access networks," Journal of Network and Systems Management, vol. 21, no. 4, pp. 677-706, 2013.

[4] C.-G. Yang, S. Trimi, and S.-G. Lee, "Effects of telecom service providers' strategic investments on business performance: a comparative study of US-Korea," Industrial Management \& Data Systems, vol. 116, no. 5, pp. 960-977, 2016.

[5] S.-L. Su, Y.-C. Tsai, and Y.-H. Yang, "A Quality-of-Service Routing Protocol with Supplementary Cooperation for Wireless Ad Hoc Networks," Wireless Personal Communications, vol. 84, no. 3, pp. 1627-1645, 2015.

[6] N. Banerjee, S. Nagar, and S. Mukherjea, "Service control layer (SCL): Enabling rule-based control and enrichment in nextgeneration telecom service delivery," Journal of Network and Systems Management, vol. 21, no. 1, pp. 65-98, 2013.

[7] K. Kimbler and M. Taylor, "Value added mobile broadband services Innovation driven transformation of the 'smart pipe", in Proceedings of the 2012 16th International Conference on Intelligence in Next Generation Networks, ICIN 2012, pp. 30-34, Germany, October 2012.

[8] Z. Yang and Z. Ma, "Analysis of communication operators transformation on smart pipe," in Proceedings of the 2013 5th International Conference on Intelligent Human-Machine Systems and Cybernetics, IHMSC 2013, pp. 131-134, China, August 2013. 
[9] H. Kashif, S. Gholamian, and H. Patel, "SLA: A stage-level latency analysisfor real-time communicationin a pipelined resource model," IEEE Transactions on Computers, vol. 64, no. 4, pp. 1177-1190, 2015.

[10] S. A. Sharna, M. R. Amin, and M. Murshed, "An enhancedMDP based vertical handoff algorithm for QoS support over heterogeneous wireless networks," in Proceedings of the 10th IEEE International Symposium on Network Computing and Applications, NCA 2011, pp. 289-293, USA, August 2011.

[11] A. Munir and A. Gordon-Ross, "An MDP-based dynamic optimization methodology for wireless sensor networks," IEEE Transactions on Parallel and Distributed Systems, vol. 23, no. 4, pp. 616-625, 2012.

[12] C. Lin, Y. Li, and J.-X. Wan, "Optimization approaches for QoS in computer networks: a survey," Jisuanji Xuebao/Chinese Journal of Computers, vol. 34, no. 1, pp. 1-14, 2011.

[13] C. Lin, J. X. Wan, X. D. Xiang, K. Meng, and Y. Z. Wang, "Dynamic optimization in computer systems and computer networks: models, solutions, and applications," Chinese Journal of Computers. Jisuanji Xuebao, vol. 35, no. 7, pp. 1339-1357, 2012.

[14] H. Osborn, "Symmetry relationships between crystal structures: applications of crystallographic group theory in crystal chemistry," Contemporary Physiscs, vol. 6, no. 1, pp. 97-98, 2015.

[15] J. Laane and E. J. Ocola, "Applications of symmetry and group theory for the investigation of molecular vibrations," Acta Applicandae Mathematicae, vol. 118, no. 1, pp. 3-24, 2012.

[16] A. Pérez-Ramírez, J. Guerrero Juk, R. Sanchez-Lara, J. A. TrejoSánchez, and L. de la Cruz-May, "Application of Mathematical Symmetrical Group Theory in the Creation Process of Digital Holograms," Mathematical Problems in Engineering, vol. 2017, 7 pages, 2017.

[17] H. N. Patel and D. K. Ghodgaonkar, "Application of group theory for computation reduction in microwave imaging of human breast model at $500 \mathrm{MHz}$," Progress in Electromagnetics Research M, vol. 51, pp. 165-173, 2016.

[18] J. Zhang, P. Wu, T. Yu et al., "Research of 3D pipeline model based on telecom operators," in Proceedings of 2015 International Conference on Mechanical, Electronic and Information Technology Engineering, pp. 681-682, 2015.

[19] T. Ayush, "Semester project report-topics in finite group theory," Diritto Marittimo, vol. 83, pp. 364-365, 2015. 


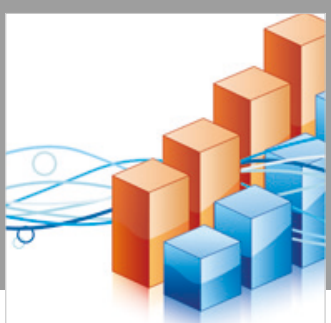

Advances in

Operations Research

\section{-n-m}
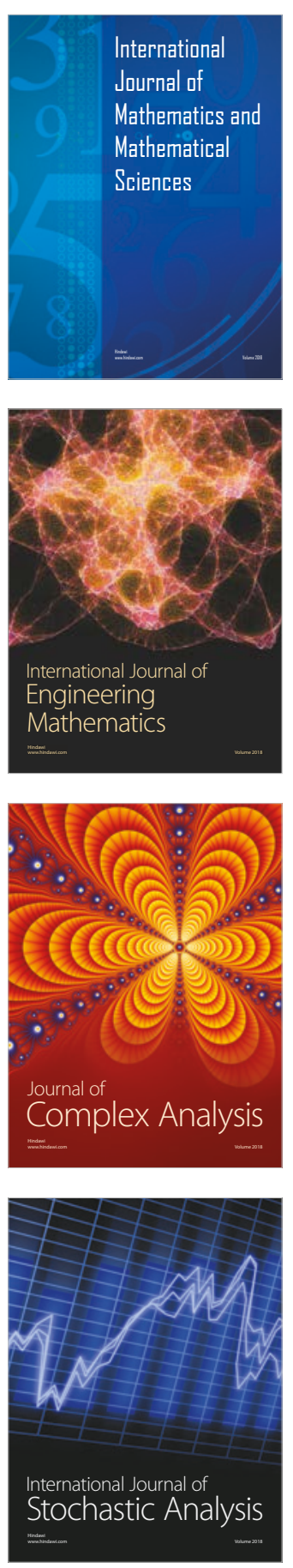
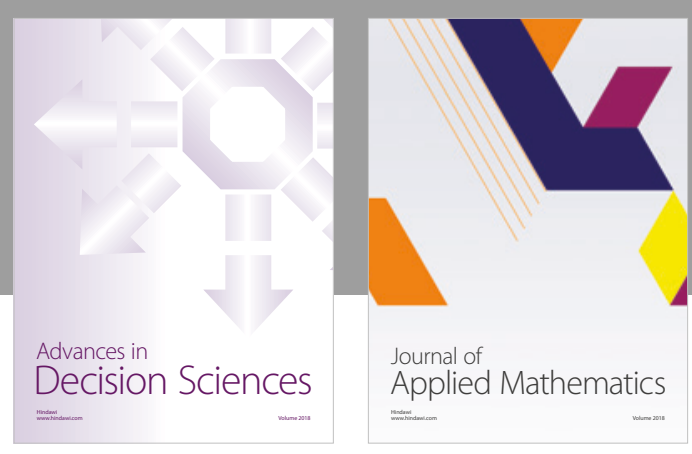

Journal of

Applied Mathematics
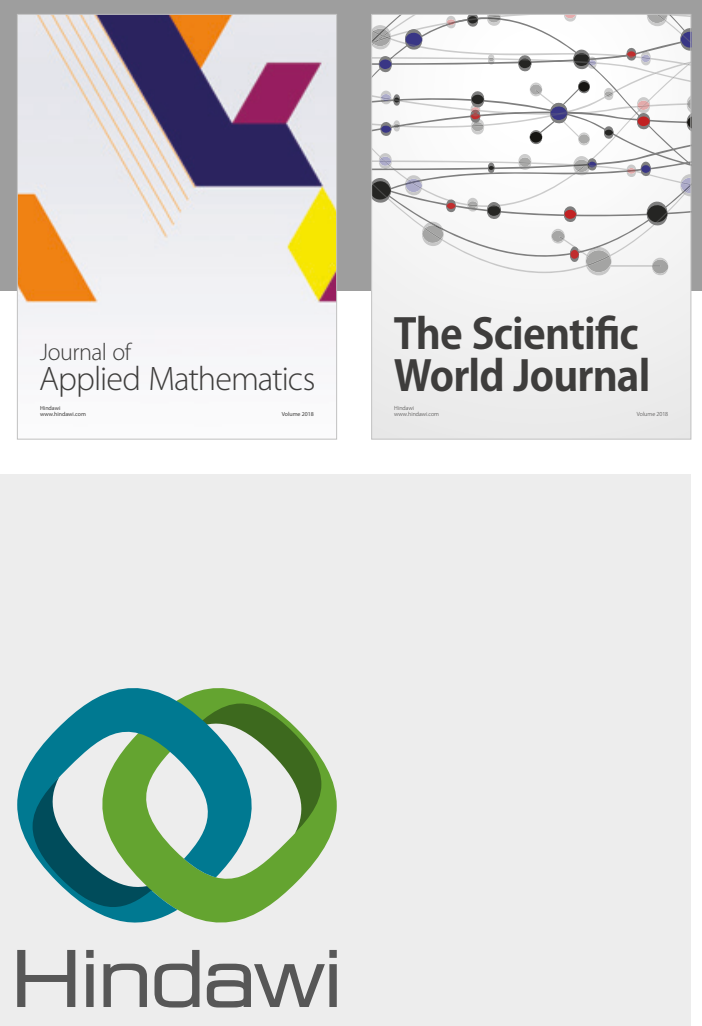

Submit your manuscripts at

www.hindawi.com

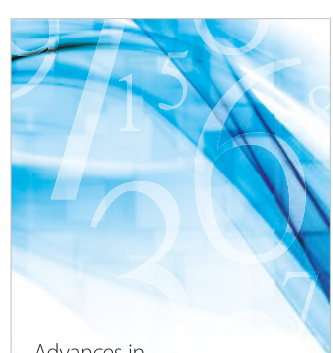

Advances in
Numerical Analysis
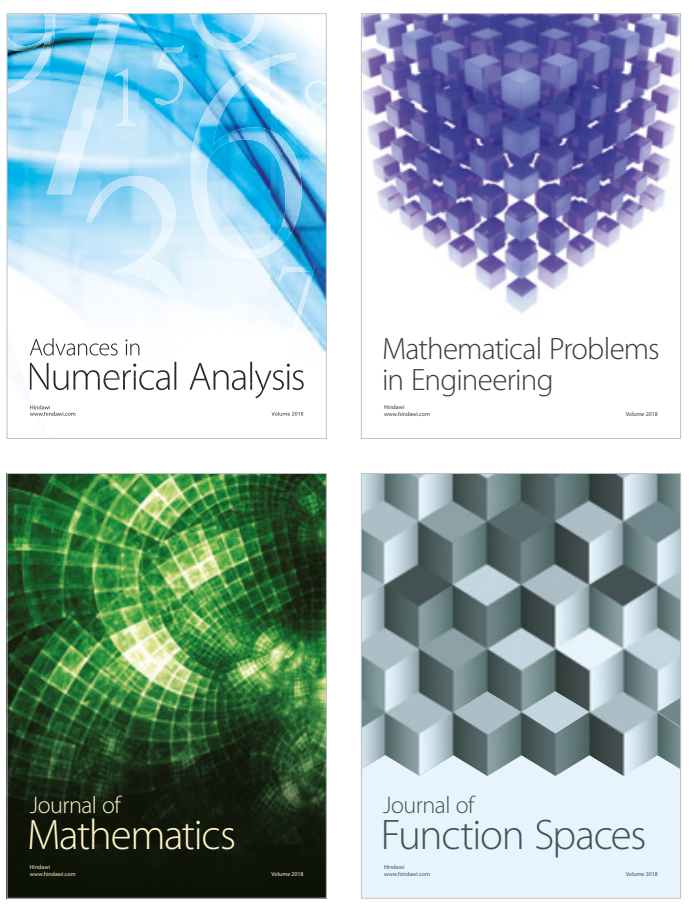

Mathematical Problems in Engineering

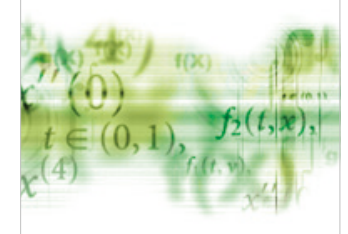

International Journal of

Differential Equations

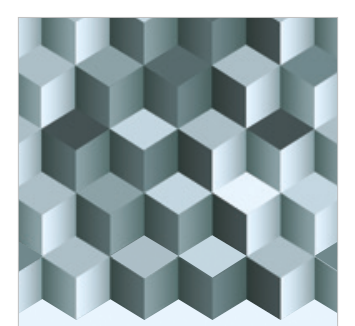

Journal of

Function Spaces

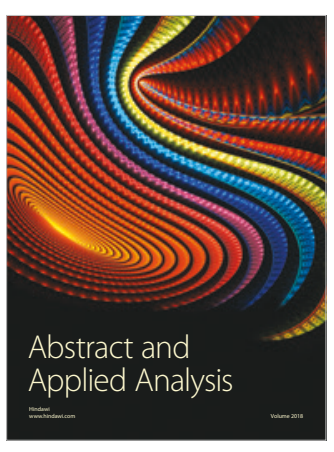

The Scientific

World Journal

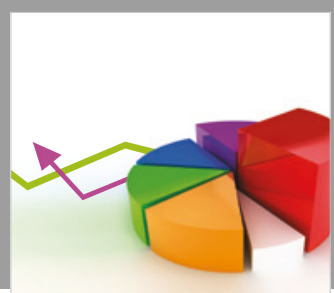

Journal of

Probability and Statistics
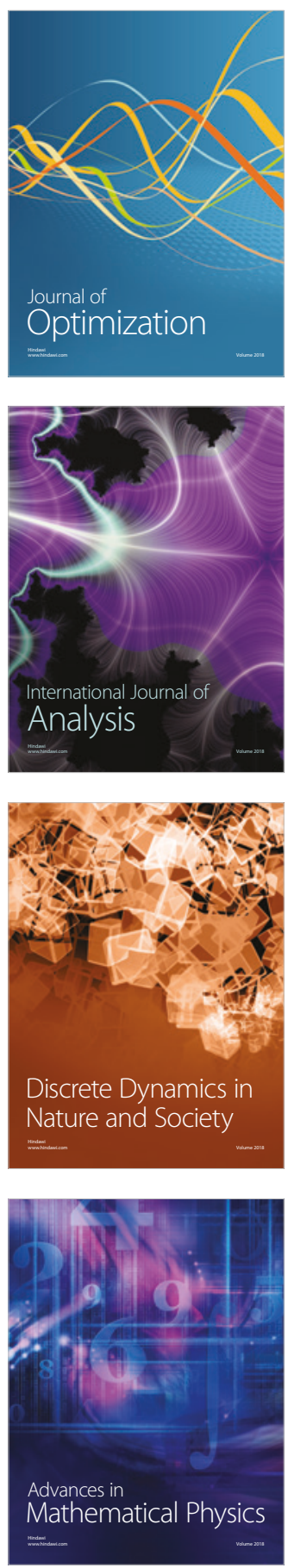